\title{
Strong Cancellation of RIN Transfer in a Raman-assisted BOTDA using Balanced Detection
}

\author{
Alejandro Dominguez-Lopez, Alexia Lopez-Gil, Sonia Martin-Lopez, Miguel Gonzalez-Herraez
}

\begin{abstract}
Raman assistance is one of the most interesting routes towards extending the range of Brillouin Optical TimeDomain Analysis (BOTDA) systems. Unfortunately, the use of Raman amplification normally entails an important relative intensity noise (RIN) transfer towards the probe wave, which severely limits this approach. In this paper, we show that the RIN transfer problem can be effectively avoided using a slightly modified scheme comprising a dual-sideband modulated probe and balanced detection. Our measurements clearly indicate a nearly complete cancellation of the RIN noise in the detected trace signal. The use of balanced detection in our Raman-assisted BOTDA setup implies a fivefold increase in Figure of Merit (FOM) compared to the same setup using conventional detection.
\end{abstract}

Index Terms- Distributed sensor, optical fiber sensors, Raman scattering, Brillouin scattering, balanced detection, temperature sensor

\section{INTRODUCTION}

B rillouin-based distributed temperature and strain fiber sensors have become strong competitors of traditional multi-point sensing systems, generating a remarkable interest for several industrial applications. Specifically, Brillouin Optical Time Domain Analysis (BOTDA) systems are increasingly used in monitoring large civil structures, such as dams, electrical grids, bridges, railways, etc.

Of course, a huge research effort is invested on extending the measuring range of these sensors. In order to do so, it is needed to increase the signal-to-noise ratio (SNR) of the system, especially when measuring in the far end of the fiber. In the past few years, several techniques have been applied trying to achieve such goal [1]-[3].

One widespread solution is the use of Raman assistance, either using first-order pumping [4] or second-order pumping [5]. Raman amplification renders a distributed gain along the fiber that allows to partially compensate the inherent fiber losses. This way, the typical exponentially decaying response of the system is avoided, and a sufficient SNR throughout the system is kept.

This work was supported in part by funding from the European Research Council through Starting Grant U-FINE (Grant no. 307441), the Spanish "Plan Nacional de I+D+i" through projects TEC2012-37958-C02-01 and TEC2012-37958-C02-02, and the INTERREG SUDOE program ECOALMGT. The work of S. Martín-López was supported by the Spanish Ministry of Science and Innovation through a "Ramón y Cajal" Contract.

A. Dominguez-Lopez, A. Lopez-Gil, S. Martin-Lopez and M. GonzalezHerraez are with Departamento de Electrónica, Universidad de Alcalá, Escuela Politécnica, 28871 Madrid, Spain (e-mail: sonia.martin@depeca.uah.es; miguel.gonzalezh@uah.es).
Despite the several advantages Raman assistance provides, it also brings Relative Intensity Noise (RIN) to the detected signal, mostly if Raman Fiber Lasers (RFL) are utilized as pumps. The spectral characteristics of the RIN introduced by the RFL is related to the resonance frequencies of the internal cavities of this type of lasers (see ref [6] and references therein). RFLs entail a relevant trade-off: they provide high power levels allowing large amplifications but also adding an important amount of RIN to the detected probe signal.

Recently, the use of balanced detection (BD) in BOTDA was demonstrated to increase the SNR of the signal by a factor of $\sqrt{ } 2$ [7]. It was also shown that a strong robustness to the deterioration of the extinction ratio of the pump pulse could be achieved.

In this paper, we demonstrate the use of $\mathrm{BD}$ in first-order Raman assisted BOTDA. We show that, on top of the already demonstrated advantages of $\mathrm{BD}$, this scheme allows effectively canceling the RIN transfer, thus improving the overall sensor characteristics.

\section{EXPERIMENTAL SETUP}

The heart of the method proposed here lies in the use of a balanced detector. In $\mathrm{BD}$, a receiver featuring two well matched photodiodes is employed. Two light beams are fed into the corresponding photodiode and the obtained currents are subtracted. Ultimately, the remaining current difference is amplified through a trans-impedance amplifier. This technique conventionally allows for the detection of small signal variations over substantial DC levels.

In order to apply BD to BOTDA systems, the probe signal is modulated with an amplitude modulator to obtain two symmetric sidebands at $\pm v_{B}$ with respect to the pump pulse frequency. Thus, stimulated Brillouin scattering (SBS) will amplify the lower frequency sideband and attenuate the higher frequency one. In detection, the sidebands are spectrally separated and fed into each detector of the BD. In typical conditions (when an external Mach-Zenhder electro-optic modulator is employed) the power of both the Stokes and Anti-Stokes bands is the same, and thus, the detected trace signal is naturally doubled in comparison with a singledetector scheme of equal responsivity [7]. Moreover, the DC component is rendered close to zero, and so the dynamic range of the detection system can be better exploited.

The benefit of the technique is clear if we now turn our attention to the signal-to-noise ratio. When the sidebands show no common-mode intensity noise, the summation of the two 


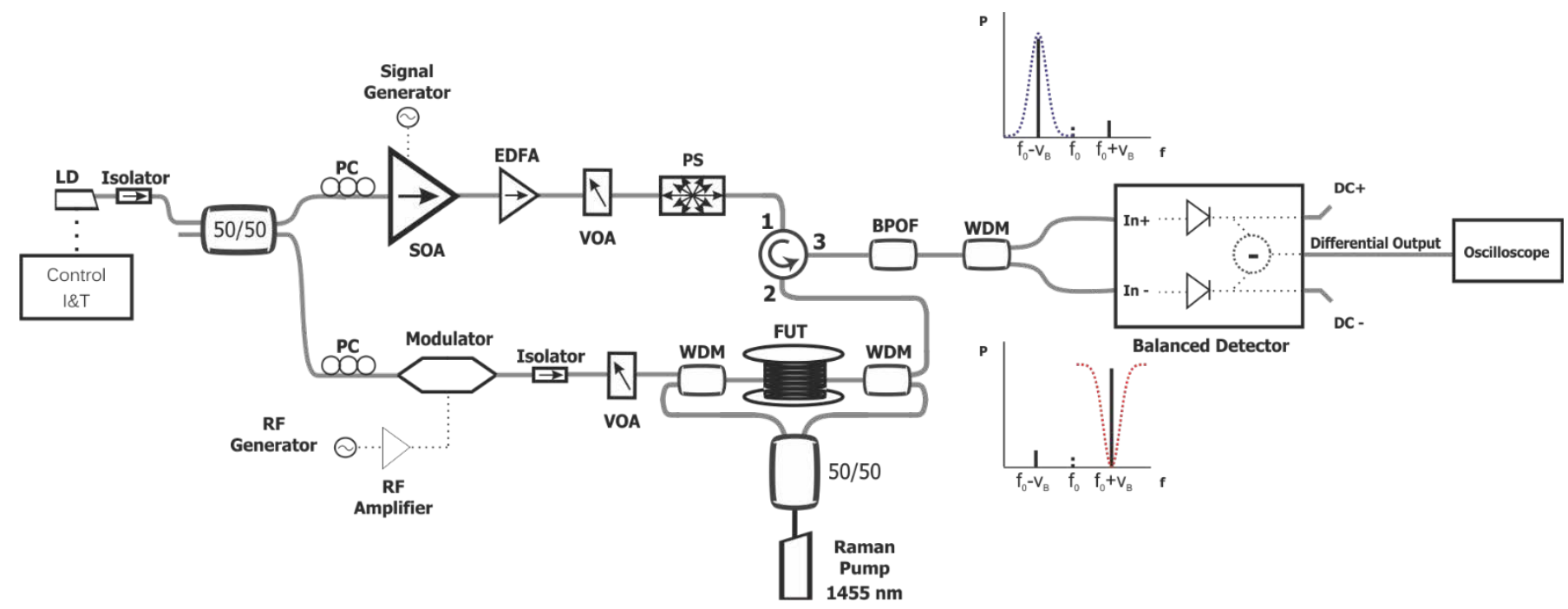

Fig. 1. Experimental setup of the first-order Raman assisted BOTDA with Balanced Detection. LD: Laser Diode; PC: Polarization controller; EDFA: Erbium Doped Fiber Amplifier; RF: Radio-frequency generator; VOA: Variable Optical Attenuator; PS: Polarization Scrambler; FUT: Fiber Under Test; WDM: Wavelength Division Multiplexer; BPOF: Band-Pass Optical Filter.

input noises coming from the two detectors (two independent equally-distributed random variables) will lead to an increase of $\sqrt{2}$ in noise. As the trace signal is doubled, the overall result is an increase of SNR of $\sqrt{ } 2$ [7]. The increase in SNR is much larger when there is any perturbation affecting both bands equally (common-mode noise). Such perturbation will be effectively cancelled by applying BD. Focusing on the particular scope of this paper, the RIN produced by the Raman pumping on the probe, will be equally found both in the upper and lower sidebands, and therefore will be eliminated upon detection.

To explore the improvement given by $\mathrm{BD}$ in Ramanassisted configurations, we have developed the scheme represented in Fig. 1. It is an evolution of a previous firstorder Raman assisted BOTDA setup [1] prepared by this group, where the detection part has been modified to include balanced detection.

As in most BOTDA setups, pump and probe waves are developed from a single distributed feed-back (DFB) laser diode. A Mach-Zehnder Electro-Optic Modulator (EOM) is used to make a dual sideband with suppressed carrier (DSBSC) modulation. The extinction ratio of the EOM is $>40 \mathrm{~dB}$, thus it is possible to nearly eliminate the carrier signal. The probe power fed into the fiber is $\sim 60 \mu \mathrm{W}$ on each sideband. The modulating frequency of the EOM is controlled through an RF Generator. The RF frequency is chosen to sweep around the Brillouin Frequency Shift of the fiber under test.

At the pump side, the signal is pulsed using a Semiconductor Optical Amplifier (SOA). The SOA allows to shape high extinction ratio $(>30 \mathrm{~dB})$ optical pulses. The pulse widths used in the experiment are $20 \mathrm{~ns}$, which implies that the sensor has 2 meter resolution. The pulses are then amplified through an Erbium Doped Fiber Amplifier (EDFA), and their power is controlled through a Variable Optical Attenuator (VOA). After the VOA, their polarization is scrambled by a fast polarization scrambler. Although this scrambler is very fast (5 $\mathrm{MHz}$ of scrambling rate), the scrambling of the polarization state is slightly imperfect, leading to some polarization noise in the trace as we will see later. The peak power of the pulses fed into the fiber is $\sim 10$ $\mathrm{mW}$.

An RFL is employed to provide first-order bi-directional Raman amplification to the system, ensuring a sufficient signal level across the fiber. The Raman pump power introduced is $\sim 240 \mathrm{~mW}$ on each propagation direction at a pump wavelength of $\sim 1455 \mathrm{~nm}$ (480 mW total power). Two WDM couplers are used to combine the Raman pumps and the Brillouin signals.

After going through the fiber and experiencing Brillouin Scattering, a band-pass optical filter (BPOF) with a 10-dB bandwidth of $2 \mathrm{~nm}$ is used to eliminate most of the amplified spontaneous emission (ASE) noise introduced by Raman amplification, leaving just the two sidebands of interest. The probe sidebands are then separated using a conventional 100 $\mathrm{GHz}$ dense wavelength division multiplexing (DWDM) filter. The edge of the filter used is sharp enough to separate both sidebands correctly, attenuating the rejected band in $>13 \mathrm{~dB}$. The Brillouin Gain band (Stokes) is fed into the positive port of the balanced detector, and Brillouin Loss band (AntiStokes) is fed into the negative port. It must be noted that effective common-mode noise cancellation will occur solely when optical lengths for both sidebands are equal before reaching the detector. Thus, care is taken to ensure that the fiber lengths covered by each sideband after separation are the same.

Ultimately, the BD system employed (Thorlabs PDB410C) will provide three different output signals: the Differential Output (with the signal of interest), and two monitoring outputs, where we will read the DC levels of each of the input signals. The sum of these DC levels will be used to normalize the detected trace and account for possible variations in the probe level [7].

It must be stressed, for comparison reasons, that this scheme is fundamentally similar to the one developed by our group in 
[1] except for the use of a balanced detection scheme.

\section{RIN CANCELLATION}

We are now ready to take a deeper look at the results obtained with the developed setup. The measurements have been performed over $\sim 100 \mathrm{~km}$ of single-mode fiber (SMF) with an essentially homogeneous Brillouin frequency shift located at $10.865 \mathrm{GHz}$ at the pump wavelength $(\sim 1550 \mathrm{~nm})$. The acquired traces have always been averaged 1024 times.

The first and most remarkable result is that, when acquiring in balanced mode, we accomplished an effective cancellation of the RFL RIN transfer. To evidence this, we performed electrical spectrum measurements of the intensity noise measured in detection, either using conventional (singlesideband) or balanced detection. Fig. 2 shows the electrical spectra of the detected probe wave acquired in both detection setups, for a total Raman pump power of $360 \mathrm{~mW}(180 \mathrm{~mW}$ per each side). In single-sideband acquisition, the RIN transfer is well visible as a set of peaks with a periodic spacing of $\sim 1.5$ $\mathrm{MHz}$. This frequency is given by the inverse of the cavity round-trip time of the primary $\mathrm{Yb}$ oscillator in the RFL. As it is visible, these RIN noise peaks are completely eliminated in the balanced detection case [8]. This is an expected result due to the fact that the RIN is common to both the Brillouin Gain (Stokes) and Brillouin Loss (anti-Stokes) bands.

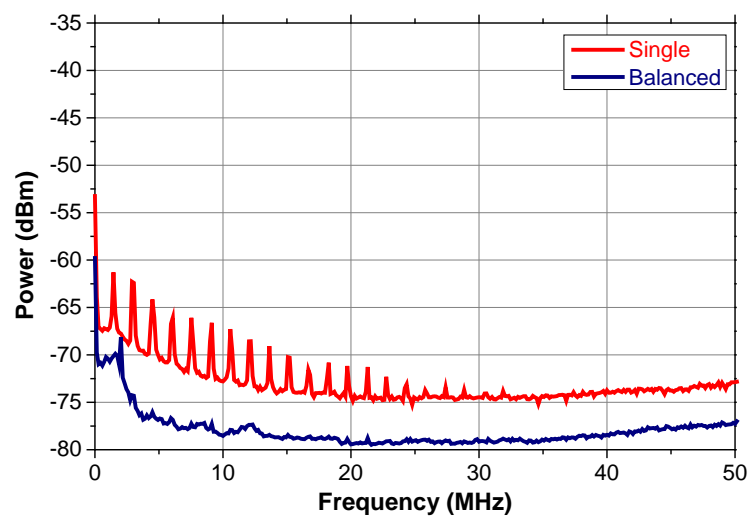

Fig. 2. Electrical spectra of the detected probe wave recorded for a total Raman pump power of $\sim 360 \mathrm{~mW}$ (30 kHz of resolution bandwidth).

To challenge the noise cancellation limits of our detection procedure, we repeated the same measurement but raising the Raman pump power to $480 \mathrm{~mW}$ ( $240 \mathrm{~mW}$ per each side). At this particular power, the RFL used brings a powerful noise peak at $44 \mathrm{MHz}$. The results are displayed in Fig. 3. As it is visible, the cancellation of the RIN noise peaks is $>15 \mathrm{~dB}$ for frequencies below $10 \mathrm{MHz}$. However, the strong peak at 44 $\mathrm{MHz}$ is not cancelled more than $10 \mathrm{~dB}$. Although a significant reduction of noise has also been achieved in this case, complete and perfect noise cancellation has not been achieved. This is due to several setup defects. First of all, the small differences in the optical path lengths $( \pm 1 \mathrm{~cm}$ in our setup) of the two single sidebands could lead to a small mismatch when arriving to the receiver, and thus, unbalance slightly the noise cancellation at high frequencies. In addition, the master laser source wavelength should be perfectly tuned in order to have exactly the same amount of probe wave detected on each port of the balanced detector. Any minimal error in the process of adjusting these parameters could lead in a non-symmetric separation of Brillouin Gain and Brillouin Loss bands previous to detection which would, once again, cause an imperfect noise cancellation. These non-idealities tend to impact the noise cancellation more severely at high frequencies. Nevertheless, high-frequency noise turns out to be more easily cancelled out in the conventional averaging procedure that is usually done in BOTDA. Hence we can safely affirm that most of the RIN transfer issues can be effectively avoided by using this configuration.

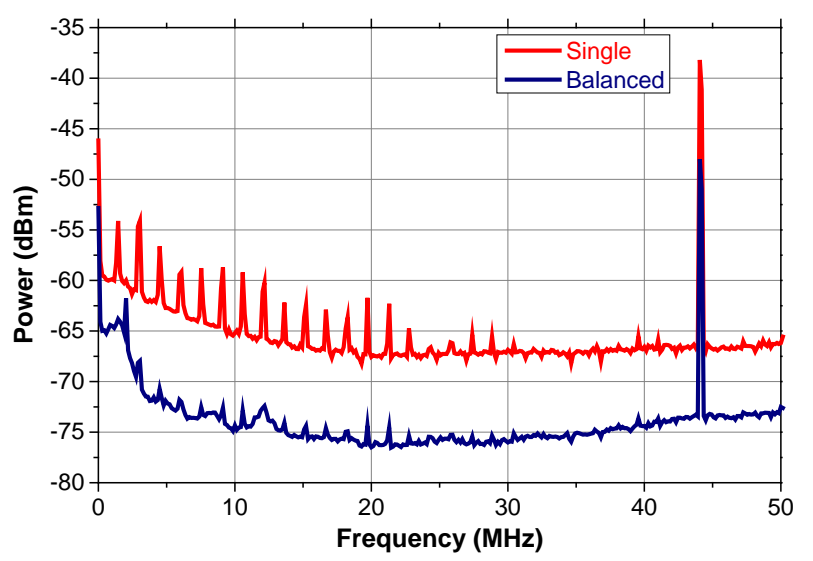

Fig. 3. Electrical spectra of the detected probe wave recorded for a total Raman pump power of $\sim 480 \mathrm{~mW}$ ( $30 \mathrm{kHz}$ of resolution bandwidth).

\section{BOTDA MEASUREMENTS}

To have a close to perfect RIN cancellation, we set the total Raman pump power back to $360 \mathrm{~mW}$. Using these settings, we performed BOTDA trace measurements over the complete length of $100 \mathrm{~km}$ with 2 meter spatial resolution (20 ns pump pulse duration).

To accomplish these BOTDA measurements, a frequency sweep is done from $10.75 \mathrm{GHz}$ to $10.95 \mathrm{GHz}$. Once the frequency sweep is finished, the Brillouin Frequency Shift (BFS) is obtained by fitting a 2nd order polynomial curve to the raw gain profile, and then finding the position of the maximum of the curve. For each frequency step, only 1024 averages are carried out. Fig. 4 shows a $~ 100 \mathrm{~km}$ amplitude trace using BD for a pump-probe frequency shift of 10.865 GHz. The trace reveals the imperfect polarization scrambling that was carried out, showing a larger polarization noise in the first kilometers of the fiber under test. The actual measured trace noise is comparable to the trace noise in other Ramanassisted BOTDA experiments over the same distance with 64 times more averaging [1].

To further verify the performance of the setup as a sensor, a hot-spot is placed in the position of worst contrast of the fiber (around $\mathrm{km} \mathrm{75).} \mathrm{This} \mathrm{is} \mathrm{done} \mathrm{by} \mathrm{immersing} 2$ meters of fiber in a hot water bath. Again, a full frequency sweep is performed to retrieve the Brillouin Frequency Shift change in 


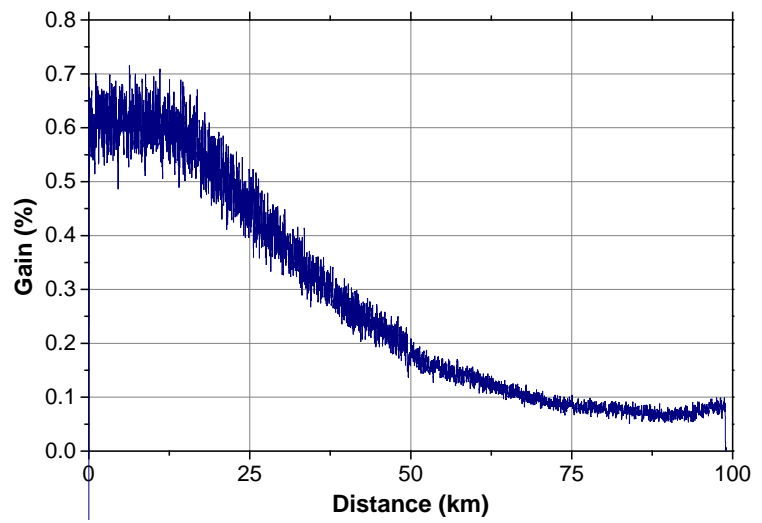

Fig. 4. First-order bi-directional Raman assisted BOTDA gain trace using BD for a pump-probe frequency shift of $10.865 \mathrm{GHz}$ and a total Raman pump power of $\sim 360 \mathrm{~mW}$.

the position of the hot-spot. The results of temperature change around the hot-spot are visible in Fig. 5. The process of translating frequency to temperature difference is rather straightforward due to the fact that the relation temperaturefrequency is linear $\left(\sim 1{ }^{\circ} \mathrm{C} / \mathrm{MHz}\right)$. A $\sim 22{ }^{\circ} \mathrm{C}$ elevation over room temperature was recorded in the hot-spot location. The system performs properly as a temperature sensor, as the temperature elevation is correctly verified with an external thermometer.

The sensing uncertainty (estimated as the standard deviation of the repeatability in 5 consecutive measurements) around the hot-spot (1000 $\mathrm{m}$ window) was $\pm 2{ }^{\circ} \mathrm{C}$. The hot spot is also correctly identified as being $\sim 2$-meter wide.

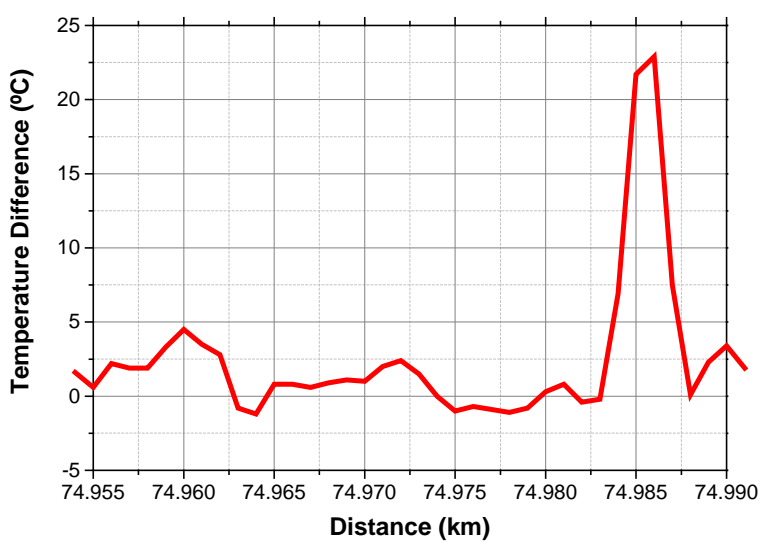

Fig. 5. Brillouin frequency shift translated to temperature difference around a 2-meter hot-spot (located around km 75).

It is interesting to compare now this setup, in terms of figure of merit (FOM) [9], with the same setup not using balanced detection [1]. While in [1] the FOM estimated was 108 , in the present setup, the FOM has been raised to 500, which implies a fivefold improvement. Therefore, on top of the expected improvement of $\sqrt{2}$ just by using balanced detection, there is an extra improvement factor of 3.5 given by the elimination of the RIN transfer. This highlights the importance of managing RIN in these Raman-assisted BOTDA systems. In terms of RIN removal, it should be noted that the FOM improvement achieved in this case is much better than when using a numerical denoising procedure [10].

\section{CONCLUSIONS}

In conclusion, we have presented a method to avoid effectively the RIN transfer problem in Raman-assisted BOTDA sensors. The method is based on using a doublesideband modulated probe (at $\pm v_{B}$ with respect to the pump pulse frequency) and balanced detection among the sidebands to profit from the push-pull effect of the gain and loss traces. The most remarkable feature of this detection scheme, with regards to the Raman-assisted configuration, is that the RIN transfer problem is effectively eliminated (as the Ramaninduced RIN is transferred to both sidebands equally). The use of balanced detection in the same Raman-assisted setup implies a fivefold increase in FOM compared to the conventional single-detector option. Moreover, we now have a clear measure of the potential improvement in FOM given by Raman assistance in any BOTDA setup, regardless of RIN noise issues.

We envisage that using this methodology, an almost zero RIN transfer scenario is feasible in most Raman-assisted BOTDA configurations (including second-order). The possible non-idealities of the setup have also been discussed (unequal optical path lengths for both Brillouin Gain and Loss bands, optimal tuning of the laser with respect to the filters, etc.).

\section{REFERENCES}

[1] X. Angulo-Vinuesa, S. Martín-López, J. Nuno, P. Corredera, J. D. AniaCastanon, L. Thevenaz, and M. Gonzalez-Herraez, "Raman-assisted Brillouin distributed temperature sensor over $100 \mathrm{~km}$ featuring $2 \mathrm{~m}$ resolution and $1.2^{\circ} \mathrm{C}$ uncertainty," IEEE J. Lightwave Technol., vol. 30, no. 8, pp. 1060-1065, 2012.

[2] M. A. Soto, P.K. Sahu, G. Bolognini, and F. Di Pasquale, "Brillouinbased distributed temperature sensor employing Pulse Coding," IEEE Sensors J., vol. 8, no. 3, pp. 225-226, 2008.

[3] M. A. Soto, M. Taki, G. Bolognini, and F. Di Pasquale, "Simplex-coded BOTDA sensor over 120-km SMF with 1-m spatial resolution assisted by optimized bidirectional Raman amplification,” IEEE Photonic. Tech. Lett., vol. 24, no. 20, pp. 1823 - 1826, 2012.

[4] F. Rodriguez-Barrios, S. Martin-Lopez, A. Carrasco-Sanz, P. Corredera, J.D. Ania-Castañon, L. Thévenaz, M. Gonzalez-Herraez, "Distributed Brillouin Fiber Sensor Assisted by First-order Raman Amplification,” J. Lightwave Technol. 28, pp. 2162-2172, 2010.

[5] S. Martin-Lopez, M. Alcon-Camas, F. Rodriguez-Barrios, P. Corredera, J.D. Ania-Castañon, L. Thévenaz, and M. Gonzalez-Herraez, "Brillouin optical time-domain analysis assisted by second-order Raman amplification”, Opt. Express 18, pp. 18769-18778, 2010.

[6] M. Krause, S. Cierullies, H. Renner, and E. Brinkmeyer "Pump-toStokes RIN transfer in Raman fiber lasers and its impact on the performance of co-pumped Raman amplifiers", Optics Communications, vol. 260, no. 2, pp. 656-661, 2006.

[7] A. Domínguez-López, A. López-Gil, S. Martín-López, and M. González-Herráez,”Signal-to-noise ratio improvement in BOTDA using balanced detection”, IEEE Photonic. Tech. Lett., vol. 26, no. 4, pp. 338341, 2014.

[8] A. Dominguez-Lopez, A. Lopez-Gil, S. Martin-Lopez, and M. Gonzalez-Herraez, "Balanced detection in Brillouin optical time domain analysis”, Proc. SPIE, vol. 9157, no. 915765, 2014.

[9] M. A. Soto, and L. Thévenaz, "Modeling and evaluating the performance of Brillouin distributed optical fiber sensors" Opt. Express, vol. 21, no. 25, pp. 31347-31366, 2013.

[10] X. Angulo-Vinuesa, S. Martín-López, P. Corredera, and M. GonzalezHerraez, "Raman-assisted Brillouin optical time-domain analysis with sub-meter resolution over $100 \mathrm{~km}$," Opt. Express, vol. 20, no. 11, pp. 12147-12154, 2012. 